\title{
The testing of saliva samples for HIV-1 antibodies: reliability in a non-clinic setting
}

\author{
A J Hunt, J Connell, G Christofinis, J V Parry, P Weatherburn, F C I Hickson, \\ A P M Coxon, P M Davies, T J McManus, S Sutherland
}

Dulwich Hospital PHLS, London G Christofinis S Sutherland

Department of Genito-Urinary Medicine, King's College Hospital, London

T J McManus

Department of Social Sciences, South Bank University, London A J Hunt

P Weatherburn

F C I Hickson

Department of

Sociology, University of Essex, Colchester A P M Coxon P M Davies

Virus Reference Laboratory, Central Public Health Laboratory, PHLS

J Connell

J V Parry

Address for correspondence A J Hunt, Project SIGMA, Department of Social Sciences, South Bank Road, London SE1 OAA, UK.

Accepted for publication 2 September 1992

\begin{abstract}
Aims-To assess the reliability of saliva samples as a means of testing for HIVantibodies outside clinic settings.

Methods-Men taking part in a nonclinic longitudinal study of homosexually active men provided samples of saliva and blood. Sera were screened using a competitive ELISA (Wellcozyme) and positive sera were confirmed by an indirect ELISA (Abbott). Saliva samples were screened either using an IgG captive radioimmunoassay or an amplified ELISA.

Results-A total of 534 paired saliva and blood samples were tested. Overall sensitivity was $96.2 \%$ and specificity was $100 \%$. None of the saliva tests were falsely positive for HIV-1 antibodies.

Conclusions-HIV-1 saliva tests can reliably be used in a non-clinic or field setting. However, if results are to be given to respondents, it is necessary to offer adequate counselling and consider the mechanisms for referral and follow-up for those that are found to be HIV-1 antibody positive.
\end{abstract}

(Genitourin Med 1993;69:29-30)

Measurements of the prevalence of HIV-1, an essential element in the epidemiological study of the spread of HIV-1, are reliably made from samples of blood. In the setting of the clinic or hospital environment this presents few problems, at least for the phlebotomist. However, many epidemiological studies of marginalised groups, such as male and female sex workers or injecting drug users, do not have access to phlebotomy services, because much field work is carried out outside of a clinic setting. Consequently, the application of a non-invasive method for HIV testing is desirable. Equally importantly some people find the giving of blood traumatic and may refuse to co-operate in the study. With these points in mind saliva tests for HIV-1 antibodies have been developed. ${ }^{12}$ The Public Health Laboratory Service has reported results from a clinical trial of the saliva test. ${ }^{3}$ To validate fully the saliva test in the nonclinic environment it needs to be shown that saliva samples can be collected by nonmedical personnel and that the saliva samples collected correspond with the blood tests.

Project SIGMA was set up in 1987 to study the lifestyles of gay and bisexual men using a non-clinic cohort. The project con- sists of a set of inter-related studies, the largest of which is a 6 year, five wave, twocentre non-clinic cohort study monitoring HIV-1 seroprevalence and changes in sexual behaviour. ${ }^{4-7}$

In this paper we present an analysis of HIV-1 antibody tests carried out on pairs of blood and saliva samples collected by non medical personnel, in the homes of respondents or at the offices of Project SIGMA.

\section{Method}

A pool of 778 (London 508, South Wales 270) gay and bisexual men were recruited from advertisements in the gay press, and by personal contact in gay clubs and pubs, and social groups and through snowballing (that is, through the social networks of existing respondents). A total of $310(61 \%)$ in London and $215(79 \%)$ in South Wales responded to the invitation to be interviewed in the first phase. In the second phase (an average of 10 months later) 274 in London and 150 in South Wales were interviewed.

At each interview men were asked (but not required) to provide a sample of blood and at the same time a sample of saliva, for testing for HIV-1 antibodies. The saliva sample was collected using a small cotton swab on which the respondent was asked to chew for half a minute. It was then sealed in a plastic collection tube which in turn was packed into a larger plastic tube. This sample was not taken within half and hour of eating or drinking. The samples were taken by trained interviewers following counselling about the test and its implications. Respondents were given the further option of receiving the result of the blood test, in which case further counselling was provided by trained counsellors. A total of 534 pairs of blood and saliva samples were provided, 304 from the first phase and 230 from the second. Blood samples were sent by post to the laboratory at Dulwich Hospital, South London, for testing. Saliva samples were forwarded to the PHLS Virus Reference Laboratory at Colindale for testing.

Sera were screened initially by a competitive enzyme immunoassay (ELISA) for antiHIV antibody (Wellcozyme, Wellcome Diagnostics) and positively reacting sera were confirmed by an indirect ELISA (Anti-HIV, 2nd generation, Abbott). Further confirmatory tests were carried out at the HIV reference laboratory at Colindale using competitive and IgG capture in-house assays for HIV antibody. 
Table Summary table of HIV-1 antibody tests on saliva-blood pairs

\begin{tabular}{lccrr}
\hline & wave 1 & wave 2 & all tests & $\%$ \\
\hline Blood and saliva negative & 273 & 214 & 487 & $94 \cdot 6$ \\
Blood and saliva positive & 17 & 8 & 25 & $4 \cdot 8$ \\
Saliva positive blood negative & 0 & 0 & 0 & $0 \cdot 0$ \\
Saliva negative blood positive & 1 & 0 & 1 & $0 \cdot 2$ \\
Equivocal & 2 & 0 & 2 & $0 \cdot 4$ \\
Total tested & 293 & 222 & 515 & $100 \cdot 0$ \\
Insufficient sample & 11 & 230 & 534 & \\
total collected & 304 & & 8 & \\
\hline
\end{tabular}

« 2 tests were equivocal, that is the saliva result for 2 positive bloods could not exclude a positive result

The saliva samples collected in wave 1 were screened by an IgG capture radioimmunoassay. For wave 2 a newly developed amplified ELISA (GACELISA) was used for screening saliva samples and GACRIA was reserved for supplementary testing. ${ }^{8}$

\section{Results}

Of the 534 pairs of blood and saliva, a comparison has been made on 515 of them (table). Saliva samples of $19(3.6 \%)$ pairs could not be tested as the sample supplied was insufficient.

For all tests the senstivity was $96 \cdot 2 \%$ and specificity $100 \%$. Using only results from the first phase sensitivity was $94.5 \%$ and specificity $100 \%$; for wave 2 sensitivity and specificity were both $100 \%$. Two saliva tests from wave 1 were equivocal, that is the saliva results for two seropositive subjects were not conclusively positive. None of the bloodsaliva pairs taken in the second phase were discordant. There were no false positive saliva results.

\section{Conclusions}

The results show that saliva collection can be successfully carried out by non-medical personnel outside of the clinic setting and is acceptable to the respondents or clients. The proportion of salivas collected where a test could not be carried out because there was an insufficient sample is not high and could be lowered by more regular training and possibly by introducing simpler saliva collection devices.

The results presented show that a saliva test for HIV-1 antibodies is a reliable alternative to a blood based test. The saliva test failed in only $3(0.6 \%)$ cases to predict accurately the blood result. All of these cases were in the first wave of collection. None of the tests carried out in wave two were inaccurate reflecting the improvements in the reliability and sensitivity of testing accompanying the introduction of the amplified ELISA.

However, it is important to recognise that if HIV-1 saliva test results are to be given to respondents then counselling before and after testing is as important as if blood sampling were used. Adequate counselling back-up is of the utmost importance as are a mechanism for referral and follow up for those who are found to be positive. Saliva testing also raises the issue of HIV transmission through exchange of saliva (especially deep kissing) as many men assumed that because antibodies could be detected then transmission could occur via saliva. Problems this may cause need to be addressed in the training of field staff who are to administer the saliva tests.

The main drawback of exclusive saliva testing is that while it is possible to carry out other serological tests, such as for hepatitis antibodies, it is not a reliable method for identifying hepatitis B carriers. However, as a general HIV-1 surveillance procedure for non-clinic groups, it does provide an alternative to the invasive procedure of taking blood.

While the research described in this paper was funded by the Medical Research Council any views expressed are those of the authors only.

1 Parry JV. An immunoglobin G capture assay (GACRIA) for anti-HTLV III/LAV and its use as a confirmatory test. $\mathcal{F} M$ Virol 1986;19:387-397.

2 Parry JV, Perry KR, Mortimer PP. Sensitive assays for viral antibodies in saliva; an alternative to tests on serum. Lancet 1987;ii:72-5.

3 Johnson AM, Parry JV, Best SJ, Smith AM, De Silva M Mortimer PP. HIV surveillance by testing saliva. AIDS 1988;2:369-71

4 Hunt AJ, Christofinis G, Coxon APM, et al. Seroprevalence of HIV-1 infection in a cohort of homosexually active men. Genitourin Med 1990;66:423-7.

5 Hunt AJ, Coxon APM, Davies PM, Weatherburn P, McManus TJ. 'Changes in sexual behaviour in a large cohort of homosexually active men in England and cohort of homosexually active men in

Wales, 1988-1989' BMF 1991;302:505-6.
Hunt AJ, Weatherburn P, Davies PM, Coxon APM Hunt AJ, Weatherburn P, Davies PM, Coxon APM,
McManus TJ. 'Sexual partners, penetrative sexual partners and HIV risk' AIDS, 1991;5: 723-8.

7 Hunt, AJ, Davies, PM, McManus TJ, et al. 'HIV-1 seroconversions in a five year non-clinic cohort of gay and bisexual men' BMF 1992;305:561-2.

8 Connell JA, Parry JV, Mortimer PP, et al. Accurate assays for anti-HIV in urine Lancet 1990;i:1366-9. 